\title{
Cohomologies of Lie Algebras of Vector Fields with Coefficients in Adjoint Representations Foliated Case
}

By

\section{Yukihiro KANIE*}

\section{Introduction}

Let $(M, \mathscr{F})$ be a foliated manifold. We have a natural Lie algebra $\mathscr{L}(M, \mathscr{F})$ of vector fields locally preserving the foliation $\mathscr{F}$, and its ideal $\mathscr{T}(M, \mathscr{F})$ of vector fields tangent to leaves of $\mathscr{F}$. Here we are interested in the first cohomologies of $\mathscr{L}(M, \mathscr{F})$ and $\mathscr{T}(M$, $\mathscr{F})$ with coefficients in their adjoint representations. This work is in a series of F. Takens' work [7] and the author's [3], [4]. In this paper, we use the latter for the general reference.

Our main result is

Main Theorem (i ) $H^{1}(\mathscr{L}(M, \mathscr{F}) ; \mathscr{L}(M, \mathscr{F}))=0$.
(ii) $H^{1}(\mathscr{T}(M, \mathscr{F}) ; \mathscr{T}(M, \mathscr{F})) \cong \mathscr{L}(M, \mathscr{F}) / \mathscr{T}(M, \mathscr{F})$.

If $M$ is compact, $\mathscr{L}(M, \mathscr{F})$ is identical with the Lie algebra of vector fields preserving $\mathscr{F}$. There are compact foliated manifolds $(M, \mathscr{F})$ such that $H^{1}(\mathscr{T}(M, \mathscr{F}) ; \mathscr{T}(M, \mathscr{F}))$ are of dimension $r$ for any $r(0 \leqq r \leqq \infty)$.

The content of this paper is arranged as follows. In $\S 1$, we introduce Lie algebras $\mathscr{L}$ and $\mathscr{T}$ for a standard foliation on a eucildean space, and study their structures. In $\S 2$, we investigate properties of derivations of $\mathscr{L}$ and $\mathscr{T}$, and in $\S 3$, we prove Main Theorem for $\mathscr{L}$ and $\mathscr{T}$ (flat case). In $\S 4$, we give the proof of Main Theorem and

Communicated by N. Shimada, June 1, 1977.

* Department of Mathematics, Mie University, Kamihama-cho, Tsu 514, Japan. 
some examples.

All manifolds, foliations, vector fields, etc. are assumed to be of $C^{\infty}$-class, throughout this paper.

\section{§ 1. Lie Algebras $\mathscr{L}$ and $\mathscr{T}$}

1. 1. Notations and Definitions. Fix a coordinate system $x_{1}, \ldots$, $x_{p}, y_{1}, \ldots, y_{q}$ in a $(p+q)$-dimensional euclidean space $V=\boldsymbol{R}^{p+q}$. Denote $\frac{\partial}{\partial x_{i}}$ by $\partial_{i}(i=1, \ldots, p)$, and $\frac{\partial}{\partial y_{\alpha}}$ by $\partial_{\alpha}(\alpha=1, \ldots, q)$. Use Latin indices $i, j, k, \ldots$ for $x_{1}, \ldots, x_{p}$, and Greek indices $\alpha, \beta, \ldots$ for $y_{1}, \ldots, y_{q}$, otherwise stated. Put

$$
\begin{aligned}
& \mathscr{T}=\left\{\sum_{i=1}^{p} f_{i}(x, y) \partial_{i} ; f_{i}(x, y) \text { are } C^{\infty} \text {-f unctions of } x_{1}, \ldots, x_{p}, y_{1}, \ldots, y_{q}\right\}, \\
& \mathscr{L}^{\prime}=\left\{\sum_{\alpha=1}^{q} g_{\alpha}(y) \partial_{\alpha} ; g_{\alpha}(y) \text { are } \mathrm{C}^{\infty} \text {-f unctions of } y_{1}, \ldots, y_{q}\right\}, \\
& \mathscr{L}=\mathscr{T}+\mathscr{L}^{\prime} \quad \text { (as vector spaces). }
\end{aligned}
$$

Then they are subalgebras of the Lie algebra $\mathfrak{A}$ of all vector fields on $V$, and $\mathscr{T}$ is an ideal of $\mathscr{L}$.

Let $\mathscr{F}$ be a standard codimension-q foliation, defined by parallel p-planes : $y_{1}=$ constant, $\ldots, y_{q}=$ constant, in $V$. Any vector field $X$ in $\mathscr{T}$ is tangent to leaves of $\mathscr{F}$, and $X$ is called leaf-tangent. Let $\phi_{t}$ be the one-parameter group of diffeomorphisms generated by $Y \in \mathscr{L}$, then $\phi_{t}$ transforms every leaf to some leaf for each $t$, and $Y$ is called foliation preserving.

Denote by $\mathscr{T}_{x}$ or $\mathscr{T}_{y}$, the subalgebra of $\mathscr{T}$ of all vector fields in $\mathscr{T}$ whose coefficient functions depend only on $x_{1}, \ldots, x_{p}$, or $y_{1}, \ldots$, $y_{q}$, respectively.

Here we summarize the facts which will be applied later.

Lemma 1. 1. ( $i$ ) Let $X \in \mathfrak{A}$. If $\left[\partial_{i}, X\right]=0$ for all $i=1, \ldots, p$, then $X$ is independent of the variables $x_{1}, \ldots, x_{p}$.

(ii) $\left[\mathscr{T}_{x}, \mathscr{L}^{\prime}\right]=0$, and $\left[\mathscr{T}_{x}, \mathscr{L}\right] \subset \mathscr{T}$.

(iii) Let $X \in \mathscr{L}$. If $\left[\partial_{i}, X\right] \in \mathscr{L}^{\prime}$ for all $i$, then $X$ is independent of the variables $x_{1}, \ldots, x_{p}$. 
(iv) Let $X \in \mathscr{T}_{y^{*}}$ Then $[X, I]=X$, where $I=\sum_{i=1}^{p} x_{i} \partial_{i} \in \mathscr{T}_{x}$.

(v) Let $X \in \mathscr{L}^{\prime}$. If $\left[X, y_{\alpha} \partial_{i}\right]=0$ for all $i$ and $\alpha$, then $X=0$.

This can be proved by elementary calculations.

1. 2. Vector Fields with Polynomial Coefficients. The vector field $X=\sum_{i=1}^{p} f_{i}(x, y) \partial_{i}+\sum_{\alpha=1}^{q} g_{\alpha}(x, y) \partial_{\alpha}$ on $V$ is said to be with polynomial coefficients, if all $f_{i}(x, y)$ and $g_{\alpha}(x, y)(i=1, \ldots, p, \alpha=1, \ldots, q)$ are polynomials. Such vector fields form a Lie subalgebra $\widetilde{\mathfrak{A}}$ of $\mathfrak{A}$. Put $\tilde{\mathscr{T}}=\mathscr{T} \cap \tilde{\mathfrak{A}}, \tilde{\mathscr{L}}=\mathscr{L} \cap \widetilde{\mathfrak{A}}$, and $\tilde{\mathscr{L}}^{\prime}=\mathscr{L}^{\prime} \cap \widetilde{\mathfrak{A}}$. Put

$$
\begin{aligned}
\mathscr{T}_{n, m}= & \left\{\sum_{i=1}^{p} f_{i}(x, y) \partial_{i} \in \tilde{\mathscr{T}} ; f_{i}(x, y)\right. \text { are homogeneous polynomials of } \\
& \text { degree } \left.n+1 \text { in } x_{1}, \ldots, x_{p}, \text { and of degree } m+1 \text { in } y_{1}, \ldots, y_{q}\right\} .
\end{aligned}
$$

Then

$$
\begin{aligned}
& \tilde{\mathscr{T}}=\sum_{n, m \geqq-1} \mathscr{T}_{n m} ; \\
& \widetilde{\mathscr{T}} \tilde{\mathscr{T}}_{x}=\tilde{\mathscr{T}} \cap \mathscr{T}_{x}=\sum_{n \geqq-1} \mathscr{T}_{n,-1}, \\
& \tilde{\mathscr{T}}_{y}=\tilde{\mathscr{T}} \cap \mathscr{T}_{y}=\sum_{m \geqq-1} \mathscr{T}_{-1, n} .
\end{aligned}
$$

Moreover, we have easily

Lemma 1.2. (cf. [4]) Let I be defined in Lemma 1.1 (iv), then $\mathscr{T}_{n,-1}=\left\{X \in \widetilde{\mathscr{T}}_{x} ;[I, X]=n X\right\}$.

Put $\mathscr{L}_{m}^{\prime}=\left\{\sum_{\alpha=1}^{q} g_{\alpha}(y) \partial_{\alpha} \in \tilde{\mathscr{L}}^{\prime} ; g_{\alpha}(y)\right.$ are homogeneous of degree $\left.m+1\right\}$. Then $\tilde{\mathscr{L}}^{\prime}=\sum_{m \geq-1} \mathscr{\mathscr { L }}_{m}^{\prime}$, and we have

Lemma 1. 3. Let $J=\sum_{\alpha=1}^{q} y_{\alpha} \partial_{\alpha} \in \mathscr{L}^{\prime}$, then $\mathscr{L}_{m}^{\prime}=\{Y \in \tilde{\mathscr{L}} ;[J, Y]=m Y\}$.

1. 3. Proposition 1. 4. If a vector field $X \in \mathscr{T}$ satisfies $j^{3}(X)(0)$ $=0$, then there exist a finite number of vector fields $X_{1}, \ldots, X_{2 r} \in \mathscr{T}$ such that

$$
X=\sum_{i=1}^{r}\left[X_{i}, X_{i+r}\right] \text { and } j^{1}\left(X_{i}\right)(0)=0(i=1, \ldots, 2 r) .
$$


Proof. Clearly it is enough to show the assertion for the case

$$
X=x_{1}^{i_{1}} \ldots \ldots x_{p}^{i_{p}} y_{1}^{j_{1}} \ldots \ldots y_{q}^{j_{q}} f(x, y) \partial_{i}
$$

for $\sum_{k=1}^{p} i_{k}+\sum_{\alpha=1}^{q} j_{\alpha} \geqq 4$. Put $h(x, y)=x_{1}^{i_{1}} \ldots x_{p}^{i_{p}} y_{1}^{j_{1}} \ldots y_{q}^{j_{q}}$.

Case 1 . The case where $i_{k} \geqq 2$ for some $k$.

$$
\begin{aligned}
& {\left[x_{k}^{2} \partial_{k}, x_{k}^{-1} X\right]-\left[x_{k}^{3} \partial_{k}, x_{k}^{-2} X\right] } \\
= & \left(i_{k}-1-2 \delta_{i k}\right) X-x_{k} h(x, y)\left(\partial_{k} f(x, y)\right) \partial_{i} \\
& -\left(i_{k}-2-3 \delta_{i k}\right) X+x_{k} h(x, y)\left(\partial_{k} f(x, y)\right) \partial_{i} \\
= & \left(1+\delta_{i k}\right) X .
\end{aligned}
$$

Here $\delta_{i k}$ is Kronecker's delta, so $\left(1+\delta_{i k}\right) \geqq 1>0$. And $j^{1}\left(x_{k}^{-2} X\right)(0)=0$ is obvious.

In the following, we can assume that $i_{k} \leqq 1$ for all $k$.

Case 2. The case where $\sum_{k} i_{k} \geqq 2$. We can assume $i_{1}=i_{2}=1$. Let $\phi$ be a coordinate transformation

$$
\phi: \begin{cases}\bar{x}_{1}=x_{1}+x_{2}, & \bar{x}_{2}=x_{1}-x_{2}, \\ \bar{x}_{i}=x_{i}(i \geqq 3), & \bar{y}_{\alpha}=y_{\alpha}(\text { all } \alpha),\end{cases}
$$

then $\phi(\mathscr{T})=\mathscr{T}$. So this case is reduced to Case 1 .

In the following, we can assume that $i_{k}=0$ for all $k$ except at most one $k_{0}$.

Case 3. The case where $j_{\alpha} \geqq 2$ for some $\alpha$. We get

$$
\left[y_{\alpha}^{2} \partial_{k_{0}}, x_{k_{0}} y_{\alpha}^{-2} X\right]-\left[y_{\alpha} x_{k_{0}} \partial_{k_{0}}, y_{\alpha}^{-1} X\right]=\left(1+\delta_{i k_{0}}\right) X .
$$

Obviously $j^{1}(Y)(0)=0$ for all vector fields $Y$ in the left hand.

Case 4. The case where $j_{\alpha} \leqq 1$ for all $\alpha$. Since we have $\sum_{\alpha=1}^{q} j_{\alpha}$ $\geqq 4-1=3$, so this case is also reduced to Case 3 , similarly as Case 2. Q. E. D.

Proposition 1.5. If a vector field $Y \in \mathscr{L}^{\prime}$ safisfies $j^{3}(Y)(0)=0$, then there exist a finite number of vector fields $Y_{1}, \ldots, Y_{2 r} \in \mathscr{L}^{\prime}$ such that 


$$
Y=\sum_{i=1}^{r}\left[Y_{i}, Y_{i+r}\right] \text { and } j^{1}\left(Y_{i}\right)(0)=0(i=1, \ldots, 2 r)
$$

Proof. Similarly as in Cases 1 and 2 in the proof of the above proposition.

Q. E. D.

\section{§ 2. Derivations of $\mathscr{T}$ and $\mathscr{L}$ ( I )}

2.1. Let $\mathscr{D}=\mathscr{D}_{e x}(\mathscr{T} ; \mathscr{L})$ be the space of derivations of $\mathscr{T}$ with values in $\mathscr{L}$. And let $\mathscr{D}_{\mathscr{L}}$ or $\mathscr{D}_{\mathscr{T}}$ be the derivation algebra of $\mathscr{L}$ or $\mathscr{T}$ respectively. Remember that a derivation $D$ satisfies the property $D[X, Y]=[D(X), Y]+[X, D(Y)]$.

Proposition 2.1. If a derivation $D$ in $\mathscr{D}$ is zero on $\mathscr{T}_{n, m}$ for $n+m \leqq-1$, then $D$ is zero on $\tilde{\mathscr{T}}$.

Proof. Step 1. To show that $D$ is zero on $\tilde{\mathscr{T}}_{x}$. We prove this by the induction on $n$ for the decomposition $\widetilde{\mathscr{T}}_{x}=\sum_{n \geq-1} \mathscr{T}_{n,-1}$. When $n$ is non-positive, the assertion holds by the assumption. Assume that $D$ is zero on $\mathscr{T}_{k,-1}(k \leqq n-1)$. Let $Z \in \mathscr{T}_{n,-1}(n \geqq 1)$, and define the vector fields $X \in \mathscr{T}$ and $Y \in \mathscr{L}^{\prime}$ as $D(Z)=X+Y$.

Apply $D$ to $\left[\partial_{i}, Z\right] \in \mathscr{T}_{n-1,-1}(1 \leqq i \leqq p)$, then we get $X \in \mathscr{T}_{y}$, by Lemma 1.1 (i) and the hypothesis of the induction.

We get $[I, Z]=n Z$, by Lemma 1.2. Apply $D$ to the both sides of this equality, then by Lemma 1.1 (iv), we get

$$
-X=n X+n Y
$$

hence $X=Y=0$, so $D(Z)=0$.

Step 2. To show that $D$ is zero on $\mathscr{T}_{0,0}$. Clearly it is enough to show the assertion for the case $X=x_{\imath} y_{\alpha} \partial_{j} \in \mathscr{T}_{0,0}$. Apply $D$ to

$$
X=x_{i} y_{\alpha} \partial_{j}=2^{-1}\left[y_{\alpha} \partial_{i}, x_{i}^{2} \partial_{j}\right],
$$

then we have $D(X)=0$, because $y_{\alpha} \partial_{i} \in \mathscr{T}_{-1,0}$ and $x_{\imath}^{2} \partial_{j} \in \tilde{\mathscr{T}}_{x}$. 
Step 3. To show that $D$ is zero on $\tilde{\mathscr{T}}_{y}$. The proof is carried out by the induction on $m$ for the decomposition $\tilde{\mathscr{T}}_{y}=\sum_{m \geq-1} \mathscr{T}_{-1, m}$. When $m$ is non-positive, the assertion holds by the assumption. Assume that $D$ is zero on $\mathscr{T}_{-1, k}(k \leqq m-1)$. Clearly it is enough to show that $D(Y)=0$ for the case

$$
Y=y_{1}^{j_{1}} \ldots \ldots y_{q}^{j_{q}} \partial_{i}
$$

for $\sum_{\alpha} j_{\alpha}=m+1$. There is an index $\beta$ such that $j_{\beta} \geqq 1$. Apply $D$ to

$$
Y=\left[y_{\beta}^{-1} Y, y_{\beta} x_{i} \partial_{i}\right],
$$

then $D(Y)=0$, because $y_{\beta}^{-1} Y \in \mathscr{T}_{-1, m-1}$, and $y_{\beta} x_{i} \partial_{i} \in \mathscr{T}_{0,0}$

Last Step. Decompose $\tilde{\mathscr{T}}$ as $\tilde{\mathscr{T}}=\sum_{n \geq-1}\left(\sum_{m \geq-1} \mathscr{T}_{n, m}\right)$. We prove the assertion of the proposition by the induction on $n$. The assertion for $n=-1$ holds by Step 3. Assume that $D$ is zero on $\sum_{m \geqq-1} \mathscr{T}_{n, m}(n \leqq$ $\left.n_{0}-1\right)$. It is enough to show that $D(X)=0$ for the case

$$
X=x_{1}^{i_{1}} \ldots \ldots x_{p}^{i_{p}} f(y) \partial_{k}
$$

for $\sum_{j} i_{j}=n_{0}+1$, and some polynomial $f(y)$ of $y_{1}, \ldots, y_{q^{*}}$ Apply $D$ to the equality

$$
X=\left\{\begin{array}{l}
\left(i_{k}+1\right)^{-1}\left[x_{k}^{-i_{k}} X, x_{k}^{i_{k}+1} \partial_{k}\right] \\
{\left[x_{k_{0}}^{-1} X, x_{k_{0}} x_{k} \partial_{k}\right]}
\end{array} \text { if } i_{k}=0, \text { and } i_{k_{0}}>0 \text { for some } k_{0},\right.
$$

we get $D(X)=0$, because $x_{k}^{-i_{k}} X, x_{k_{0}}^{-1} X \in \sum_{n \leqq n_{0}-1}\left(\sum_{m \geqq-1} \mathscr{T}_{n, m}\right)$, and $x_{k^{k}}^{i+1} \partial_{k}$, $x_{k_{0}} x_{k} \partial_{k} \in \widetilde{\mathscr{T}}_{2}$.

Q. E. D.

Corollary 2.2. The derivation $D \in \mathscr{D}$ is zero on $\mathscr{T}$, under the same assumption as Proposition 2. 1.

Proof. It follows from Propositions 1.3 and 1.4 in [4], and Proposition 1. 4.

Q. E. D.

2. 2. Proposition 2. 3. If a derivation $D \in \mathscr{D}_{\mathscr{L}}$ is zero on $\mathscr{T}$, then $D$ is zero on $\tilde{\mathscr{L}}^{\prime}$. 
Proof. Step 1. To show that $D\left(\partial_{\alpha}\right)=0(\alpha=1, \ldots, q)$. Apply $D$ to $\left[\partial_{i}, \partial_{\alpha}\right]=\left[I, \partial_{\alpha}\right]=0$, then we get $D\left(\partial_{\alpha}\right) \in \mathscr{L}^{\prime}$, by Lemma $1.1(\mathrm{i})$, (iv).

Define the functions $\mathrm{g}_{\alpha}^{\beta}(y)$ as $D\left(\partial_{\alpha}\right)=\sum_{\beta} g_{\alpha}^{\beta}(y) \partial_{\beta} \in \mathscr{L}^{\prime}$. Apply $D$ to $\delta_{\alpha r} \partial_{i}=\left[\partial_{\alpha}, y_{r} \partial_{i}\right]$, then we get

$$
0=\left[\sum_{\beta} g_{\alpha}^{\beta}(y) \partial_{\beta}, y_{r} \partial_{i}\right]=g_{\alpha}^{\gamma}(y) \partial_{i},
$$

hence $\mathrm{g}_{\alpha}^{\gamma}(y)=0$, so $D\left(\partial_{\alpha}\right)=0$.

Step 2. To show that $D(J)=0$, where $J=\sum_{\alpha=1}^{q} y_{\alpha} \partial_{\alpha}$. Apply $D$ to $\left[\partial_{i}, J\right]=\left[I, J=0\right.$, then we get $D(J) \in \mathscr{L}^{\prime}$, by Lemma 1.1 (i), (iv).

Apply $D$ to $\left[J, y_{\alpha} \partial_{i}\right]=y_{\alpha} \partial_{i} \in \mathscr{T}$, then we have $D(J)=0$, by Lemma 1.1 (v).

Last Step. Since $\tilde{\mathscr{L}}^{\prime}$ is decomposed as $\tilde{\mathscr{L}}^{\prime}=\sum_{m \geq-1} \mathscr{L}_{m}^{\prime}$ (cf. § 1.2), then by Lemma 1. 3, this step is carried out similarly as Step 1 in the proof of Proposition 2. 1.

Q. E. D.

Corollary 2.4. If a derivation $D$ of $\mathscr{L}$ is zero on $\mathscr{T}_{n, m}$ for $n$ $+m \leqq-1$, then $D$ is zero on $\mathscr{L}$.

Proof. Let $D$ be a derivation of $\mathscr{L}$ such that $D$ is zero on $\mathscr{T}_{n, m}$ for $n+m \leqq-1$. Let $D^{\prime}$ be the restriction of $D$ to $\mathscr{T}$. Then by Coroallry 2.2, $D^{\prime}$ is zero on $\mathscr{T}$, hence by Proposition $2.3, D$ is zero on $\tilde{\mathscr{L}}^{\prime}$. The assertion follows from Propositions 1.3 and 1.4 in [4] and Proposition 1.5.

Q. E. D.

\section{§ 3. Derivations of $\mathscr{T}$ and $\mathscr{L}$ (III)}

3. 1. Determination of $\mathscr{D}$. Let $Z$ be a vector field on $V$. We define $\operatorname{ad} Z$ as $\operatorname{ad} Z(X)=[Z, X]$ for $X \in \mathfrak{A}$. Then we have

Lemma 3. 1. The map: $\left.Z \longrightarrow \mathrm{ad} Z\right|_{\mathscr{T}}$, or $\left.Z \longrightarrow \mathrm{ad} Z\right|_{\mathscr{L}}$ of $\mathscr{L}$ into $\mathscr{D}$ or $\mathscr{D}_{\mathscr{L}}$ respectively is an into isomorphism. 
Proof. It is sufficient to show the injectivity. Let $Z \in \mathscr{L}$. Assume that $\operatorname{ad} Z(\mathscr{T})=0$. By Lemma 1.1 (i), we get the vector fields $X \in$ $\mathscr{T}_{y}$ and $Y \in \mathscr{L}^{\prime}$ such that $Z=X+Y$. Then by Lemma 1.1 (ii), (iv), we have $X=[Z, I]=0$, whence $Y=0$, by Lemma 1.1 (v).

Q. E. D.

Theorem 3.2. Let $D \in \mathscr{D}$. Then there exists a unique vector field $W$ on $V$ such that $D=\left.\operatorname{ad} W\right|_{\mathscr{\sigma}}$. Moreover, $W$ is in $\mathscr{L}$.

The proof of this theorem will be given in $\S 3.3$.

Corollary 3. 3. Let $D \in \mathscr{D}_{\mathscr{T}}$ or $\mathscr{D}_{\mathscr{L}}$. Then there exists a unique vector field $W \in \mathfrak{X}$ such that $D=\left.\operatorname{ad} W\right|_{\mathscr{T}}$ or $=\left.\operatorname{ad} W\right|_{\mathscr{L}}$. Moreover, $W$ is in $\mathscr{L}$.

Proof. Obvious for the case $D \in \mathscr{D}_{\mathscr{T}}$. Let $D \in \mathscr{D}_{\mathscr{L}}$. The restriction of $D$ to $\mathscr{T}$ belongs to $\mathscr{D}$. Then the assertion follows from Theorem 3. 2 and Corollary 2. 4.

Q. E. D.

Theorem 3.4. ( $i$ ) All derivations of $\mathscr{L}$ are inner, that is, $\mathscr{D} \mathscr{L}$ $=\operatorname{ad} \mathscr{L} \cong \mathscr{L}$. Hence

$$
H^{1}(\mathscr{L} ; \mathscr{L})=0 .
$$

(ii) The derivation algebra of $\mathscr{T}$ is naturally isomorphic to $\mathscr{L}$, that is, $\mathscr{D}_{\mathscr{T}}=\left\{\left.\operatorname{ad} W\right|_{\mathscr{T}} ; W \in \mathscr{L}\right\} \cong \mathscr{L}$. Hence

$$
H^{1}(\mathscr{T} ; \mathscr{T}) \cong \mathscr{L} / \mathscr{T} \cong \mathscr{L}^{\prime}
$$

In particular, the space $H^{1}(\mathscr{T} ; \mathscr{T})$ is of infinite dimension.

Proof. (ii) By Coroallry 3.3, we have $\mathscr{D}_{\mathscr{T}} \subset\left\{\left.\operatorname{ad} W\right|_{\mathscr{T}} ; W \in \mathscr{L}\right\}$. The converse inclusion is obvious, because $\mathscr{T}$ is an ideal of $\mathscr{L}$. For the latter half, remember that $H^{1}(\mathscr{T} ; \mathscr{T}) \cong \mathscr{D} \mathscr{T} /$ ad $\mathscr{T}$ (see $\S 1$ in [3]).

Q. E. D.

3. 2. To prove Theorem 3.2, we prepare the following four 
lemmata.

Lemma 3.5. Let $D \in \mathscr{D}$. Then there exists a vector field $W_{1}$ $\in \mathscr{T}$ such that $D\left(\partial_{i}\right) \equiv\left[W_{1}, \partial_{i}\right]\left(\bmod \mathscr{L}^{\prime}\right)$ for $i=1, \ldots, p$.

Proof. Define the functions $f_{i}^{j}(x, y)$, and the vector fields $Y_{i}$ $\in \mathscr{L}^{\prime}$, as

$$
D\left(\partial_{i}\right)=\sum_{j=1}^{p} f_{i}^{j}(x, y) \partial_{j}+Y_{i} \quad(1 \leqq i \leqq p) .
$$

Apply $D$ to the both sides of $\left[\partial_{i}, \partial_{k}\right]=0$, then we have, by Lemma 1.1 (ii),

$$
\sum_{j=1}^{p}\left\{\partial_{i}\left(f_{k}^{j}(x, y)\right)-\partial_{k}\left(f_{i}^{j}(x, y)\right)\right\} \partial_{j}=0 \quad(1 \leqq i, k \leqq p),
$$

and so

$$
\partial_{i}\left(f_{k}^{j}(x, y)\right)=\partial_{k}\left(f_{i}^{j}(x, y)\right) \quad(1 \leqq i, j, k \leqq p) .
$$

Therefore, there are unique functions $h^{j}(x, y)(1 \leqq j \leqq p)$ such that

$$
\begin{cases}\partial_{i}\left(h^{j}(x, y)\right)=f_{i}^{j}(x, y) & (1 \leqq i, j \leqq p) \\ h^{j}(0, y)=0 & (1 \leqq j \leqq p)\end{cases}
$$

Put $W_{1}=-\sum_{i=1}^{p} h^{i}(x, y) \partial_{i}$, then we have the assertion of the lemma.

Q. E. D.

Lemma 3.6. Let $D \in \mathscr{D}$. Assume that $D\left(\partial_{i}\right) \in \mathscr{L}^{\prime}(1 \leqq i \leqq p)$. Then (i) $D\left(\partial_{i}\right)=0 \quad(1 \leqq i \leqq p)$,

(ii) there exists a vector field $W_{2} \in \mathscr{T}$ such that $\left[\partial_{\imath}, W_{2}\right]=0$ (1 $\leqq i \leqq p)$, and $D(I) \equiv\left[W_{2}, I\right]\left(\bmod \mathscr{L}^{\prime}\right)$.

Proof. Define the vector fields $X \in \mathscr{T}$ and $Y \in \mathscr{L}^{\prime}$ as $D(I)=X$ $+Y$. Apply $D$ to $\left[\partial_{i}, I\right]=\partial_{i}$, then by Lemma 1.1 (ii), (iii), we have that $D\left(\partial_{i}\right)=0 \quad(1 \leqq i \leqq p)$, and $X \in \mathscr{T}_{y}$. Hence, by Lemma 1.1 (iv), we get

$$
[X, I]=X \equiv D(I) \quad\left(\bmod \mathscr{L}^{\prime}\right)
$$

Therefore, we can put $W_{2}=X$.

Q. E. D. 
Lemma 3.7. Let $D \in \mathscr{D}$. Assume that $D\left(\partial_{i}\right)=0 \quad(1 \leqq i \leqq p)$, and $D(I) \in \mathscr{L}^{\prime}$. Then, $D\left(x_{i} \partial_{j}\right) \in \mathscr{L}^{\prime} \quad(1 \leqq i, j \leqq p)$.

Proof. Define the vector fields $X_{i j} \in \mathscr{T}$ and $Y_{i j} \in \mathscr{L}^{\prime}$ as $D\left(x_{i} \partial_{j}\right)$ $=X_{i j}+Y_{i j}(1 \leqq i, j \leqq p)$.

Apply $D$ to $\left[\partial_{k}, x_{i} \partial_{j}\right]=\delta_{i k} \partial_{j}$, then by Lemma 1.1 (i), we have $X_{i j}$ $\in \mathscr{T}_{y}(1 \leqq i, j \leqq p)$. Apply $D$ to $\left[I, x_{i} \partial_{j}\right]=0$, then by Lemma 1.1 (ii), (iv), we get $X_{i j}=0(1 \leqq i, j \leqq p)$.

Q. E. D.

Lemma 3. 8. Let $D \in \mathscr{D}$. Assume that $D\left(\partial_{i}\right)=0$, and that $D(I)$ $\in \mathscr{L}^{\prime}, D\left(x_{i} \partial_{j}\right) \in \mathscr{L}^{\prime}(1 \leqq i, j \leqq p)$. Then,

(i) $D(I)=0, D\left(x_{i} \partial_{j}\right)=0 \quad(1 \leqq i, j \leqq p)$,

(ii) there exists a unique vector field $W_{3}$ on $V$ such that

$$
\begin{aligned}
& {\left[W_{3}, \partial_{i}\right]=\left[W_{3}, I\right]=\left[W_{3}, x_{i} \partial_{j}\right]=0,} \\
& {\left[W_{3}, y_{\alpha} \partial_{i}\right]=D\left(y_{\alpha} \partial_{i}\right) \quad(1 \leqq i, j \leqq p, 1 \leqq \alpha \leqq q) .}
\end{aligned}
$$

Moreover, $W_{3}$ is in $\mathscr{L}^{\prime}$.

Proof. Define the vector fields $X_{\alpha i} \in \mathscr{T}$ and $Y_{\alpha i} \in \mathscr{L}^{\prime}$ as $D\left(y_{\alpha} \partial_{i}\right)$ $=X_{\alpha i}+Y_{\alpha i}(1 \leqq i \leqq p, 1 \leqq \alpha \leqq q)$. Apply $D$ to $\left[\partial_{j}, y_{\alpha} \partial_{i}\right]=0$, then by Lemma 1. 1 (i), we have $X_{\alpha i} \in \mathscr{T}_{y}$ for all $i$ and $\alpha$. Apply $D$ to $y_{\alpha} \partial_{i}=\left[y_{\alpha} \partial_{i}\right.$, $I$, then by Lemma 1.1 (ii), (iv), we get that $D(I)=0$ and $Y_{\alpha i}=0$ for all $i$ and $\alpha$.

Define the functions $f_{\alpha i}^{j}(y)(1 \leqq i, j \leqq p, 1 \leqq \alpha \leqq q)$ as $\mathrm{X}_{\alpha i}=\sum_{j} f_{\alpha i}^{j}(y) \partial_{j}$. Apply $D$ to $y_{\alpha} \partial_{i}=\left[y_{\alpha} \partial_{i}, x_{i} \partial_{i}\right]$, then we get

$$
\sum_{j} f_{\alpha i}^{j}(y) \partial_{j}=f_{\alpha i}^{i}(y) \partial_{i}+\left[y_{\alpha} \partial_{i}, D\left(x_{i} \partial_{i}\right)\right],
$$

hence $D\left(x_{i} \partial_{i}\right)=0(1 \leqq i \leqq p)$, and $f_{a i}^{j}(y)=0$ for all $i \neq j$ and $\alpha$.

Apply $D$ to $y_{\alpha} \partial_{k}=\left[y_{\alpha} \partial_{i}, x_{i} \partial_{k}\right]$ for $i \neq k$, then we get

$$
f_{\alpha k}^{k}(y) \partial_{k}=f_{\alpha i}^{i}(y) \partial_{k}+\left[y_{\alpha} \partial_{i}, D\left(x_{i} \partial_{k}\right)\right],
$$

hence $D\left(x_{i} \partial_{k}\right)=0(1 \leqq i, k \leqq p)$, and $f_{a i}^{i}(y)=f_{\alpha k}^{k}(y)$ for all $i \neq k$ and $\alpha$. Denote $f_{a i}^{i}(y)$ by $f_{\alpha}(y) \quad(1 \leqq \alpha \leqq q)$, then $D\left(y_{\alpha} \partial_{i}\right)=f_{\alpha}(y) \partial_{i}$.

Let $W_{3}$ be a vector field on $V$ satisfying the equations in (ii). Since $\left[W_{3}, \partial_{i}\right]=\left[W_{3}, I\right]=0(1 \leqq i \leqq p)$, then we get $W_{3} \in \mathscr{L}^{\prime}$, by Lemma 
1. 1(i), (iv). Write $W_{3}$ as $W_{3}=\sum_{\beta} h_{\beta}(y) \partial_{\beta}$, then

$$
\left[W_{3}, y_{\alpha} \partial_{i}\right]=h_{\alpha}(y) \partial_{i} \quad(1 \leqq i \leqq p, 1 \leqq \alpha \leqq q) .
$$

Hence, $h_{\alpha}(y)$ must be equal to $f_{\alpha}(y)$ for all $\alpha$.

Q. E. D.

3. 3. Proof of Theorem 3.2. Let $D \in \mathscr{D}$. Then, by Lemmata 3.5 3. 8, we have a unique vector field $W$ on $V$ such that $D$ $=\operatorname{ad} W$ on $\mathscr{T}_{n, m}$ for $n+m \leqq-1$. We can determine $W$ as $W=W_{1}$ $+W_{2}+W_{3}$, where $W_{i}(i=1,2,3)$ are given in the above lemmata. Clearly $W \in \mathscr{L}$.

Hence, by Corollary 2. 2, we get that $D=\operatorname{ad} W$ on $\mathscr{T}$.

Q. E. D.

3.4. Remarks. ( i ) Any derivation of $\mathscr{T}$ or $\mathscr{L}$ is continuous, because it is realized as ad $W$ for some $W \in \mathscr{L}$.

(ii) Let $V^{\prime}$ be a subspace of $V$, spanned by $y_{1}, \ldots, y_{q}$. Then Theorem 3.4 (i) can be rewritten as in the following form in terms of $C^{\infty}\left(V^{\prime}\right)$, which is suggestive for calculations of cohomologies of $\mathscr{T}$ with various coefficients.

Theorem 3.9. Let $\mathscr{D}_{e x}\left(C^{\infty}\left(V^{\prime}\right)\right)$ be the derivation algebra of the associative algebra $C^{\infty}\left(V^{\prime}\right)$. Then

$$
H^{1}(\mathscr{T} ; \mathscr{T}) \cong \mathscr{D}_{\text {ex }}\left(C^{\infty}\left(V^{\prime}\right)\right)
$$

This follows immediately from the following well-known fact.

Lemma 3.10. There is an natural Lie algebra isomorphism of $\mathscr{L}^{\prime}$ onto $\mathscr{D} \operatorname{ex}\left(C^{\infty}\left(V^{\prime}\right)\right)$.

We give here its elementary proof for completeness. Let $D \in \mathscr{D}_{e x}$ $\left(C^{\infty}\left(V^{\prime}\right)\right)$. Define functions $g_{\alpha}(y)(\alpha=1, \ldots, q)$ as $D\left(y_{\alpha}\right)=g_{\alpha}(y)$. Let $Y=\sum_{\alpha} g_{\alpha}(y) \partial_{\alpha} \in \mathscr{L}^{\prime}$. The vector field $Y$ operates on $C^{\infty}\left(V^{\prime}\right)$ as a first-order partial differential operator, then it defines a derivation 
$D_{Y}$ of $C^{\infty}\left(V^{\prime}\right)$. Easily by induction, we can show that $D$ coincides with $D_{Y}$ on the polynomial algebra $\mathbb{R}\left[y_{1}, \ldots, y_{q}\right]$. Hence we obtain Lemma 3. 10, because when $j^{2}(g)(0)=0, g$ is expressed as $g(y)$ $=\sum_{\alpha, \beta} y_{\alpha} y_{\beta} g_{\alpha \beta}(y)$ with $g_{\alpha \beta} \in C^{\infty}\left(V^{\prime}\right)$.

\section{§ 4. Lie Algebras $\mathscr{L}(\mathbf{M}, \mathscr{F}), \mathscr{T}(\mathbf{M}, \mathscr{F})$, and Their Derivations}

4. 1. Lie Algebras Associated with Foliations. Let $M$ be a $(p$ $+q$ )-dimensional manifold and $\mathscr{F}$ a codimension- $q$ foliation on $M$. Around any point of $M$, there is a distinguished coordinate neighborhood $\left(U ; x_{1}, \ldots, x_{p}, y_{1}, \ldots, y_{q}\right)$, for which a plate represented as $y_{1}$ constant,..., $y_{q}=$ constant in $U$ is a connected component of $L \cap U$ for some leaf $L$ of $\mathscr{F}$ (see e.g. [6] for definitions).

A vector field $X$ on a foliated manifold $(M, \mathscr{F})$ is called leaftangent, if $X$ is tangent to the leaf $L$ through $p$ for any point $p$ of $M$, that is, the vector $X_{p}$ belongs to the tangent space $\mathrm{T}_{p} L$ of $L$ at p. $A$ vector field $X$ is called to be locally foliation preserving (or $l$. $f . p_{.}$, in short), if $\phi_{t}$ maps every plate to some plate, where $\left\{\phi_{t}\right\}$ is a one-parameter group of local diffeomorphisms generated by $X$.

Locally for any distinguished coordinates $\left(x_{1}, \ldots, x_{p}, y_{1}, \ldots, y_{q}\right)$, a leaf-tangent vector field is represented as $\sum_{i=1}^{p} f_{i}(x, y) \partial_{i}$, and a $l . f$. p. vector field is represented as $\sum_{i=1}^{p} f_{i}(x, y) \partial_{i}+\sum_{\alpha=1}^{q} g_{a}(y) \partial_{\alpha}$, where $f_{i}(x$, y) $(i=1, \ldots, p)$ are $C^{\infty}$-functions of $x_{1}, \ldots, x_{p}, y_{1}, \ldots, y_{q}$, and $g_{\alpha}(y)$ $(\alpha=1, \ldots, q)$ are $C^{\infty}$-functions of $y_{1}, \ldots, y_{q}$. Here we use the notations $\partial_{i}$ or $\partial_{\alpha}$ instead of $\frac{\partial}{\partial x_{i}}$ or $\frac{\partial}{\partial y_{\alpha}}$ respectively, and the convention on indices (see $\S 1.1$ ).

All $l . f . p$. vector fields on $(M, \mathscr{F})$ form a Lie algebra $\mathscr{L}(M, \mathscr{F})$, and all leaf-tangent vector fields form its ideal $\mathscr{T}(M, \mathscr{F})$.

If a $l . f . p$. vector field $X$ is complete, then $X$ is foliation preserving, that is, the diffeomorphism $\phi_{t}$ maps every leaf of $\mathscr{F}$ to some leaf for each t. Simliarly, if a leaf-tangent vector field $X$ is complete, $\phi_{t}$ leaves every leaf of $\mathscr{F}$ stable. Thus, when $M$ is compact, $l . f . p$. vector fields are foliation preserving. 
4. 2. Derivations. Let $\mathscr{D}(M, \mathscr{F})=\mathscr{D}$ ex $(\mathscr{T}(M, \mathscr{F}) ; \mathscr{L}(M, \mathscr{F}))$ be the space of derivations of $\mathscr{T}(M, \mathscr{F})$ with values in $\mathscr{L}(M, \mathscr{F})$. And let $\mathscr{D}_{\mathscr{L}}(M, \mathscr{F})$ or $\mathscr{D}_{\mathscr{T}}(M, \mathscr{F})$ be the derivation algebra of $\mathscr{L}(M, \mathscr{F})$ or $\mathscr{T}(M, \mathscr{F})$ respectively. Sometimes we omit $\mathscr{F}$ in the notations $\mathscr{T}(M, \mathscr{F}), \mathscr{D}(M, \mathscr{F})$, etc.

Lemma 4.1. Let $U$ be an open subset of $M$, and $X \in \mathscr{L}(M, \mathscr{F})$. Assume that $[X, Y]=0$ on $U$ for any $Y \in \mathscr{T}(M, \mathscr{F})$ with support contained in $U$. Then, $X=0$ on $U$.

Proof. Let $p \in U$. Take a sufficiently small neighborhbood $U^{\prime}$ of $p$ in $U$, and distinguished coordinates $\left(x_{1}, \ldots, x_{p}, y_{1}, \ldots, y_{q}\right)$ in $U^{\prime}$. Let a vector field $Y^{\prime}$ on $U^{\prime}$ be any one of $\partial_{\imath}, x_{j} \partial_{\imath}$, and $y_{\alpha} \partial_{i}(1 \leqq i, j$ $\leqq p, 1 \leqq \alpha \leqq q)$. Since $\mathscr{T}(M)$ is $C^{\infty}(M)$-module, there is a vector field $Y \in \mathscr{T}(M)$ such that $Y=Y^{\prime}$ on $U^{\prime}$ and the support of $Y$ is contained in $U$. Then we have $[X, Y]=0$ on $U$ by the assumption. By the proof of Lemma 3.8, we have that $X=0$ on $U^{\prime}$, in particular, at $p$. Hence we get $X=0$ on $U$.

Q. E. D.

From this lemma, we get the following two lemmata, similarly as Proposition 2.4 and Corollary 2.5 in [4].

Lemma 4.2. Let $D \in \mathscr{D}(M, \mathscr{F})$ or $\mathscr{D} \mathscr{L}(M, \mathscr{F})$. Then, $D$ is local.

Lemma 4. 3. Let $D \in \mathscr{D}(M, \mathscr{F})$. Then, $D$ is localizable (see $\S 1.2$ in [4] for definition).

4. 3. Proposition 4. 4. Let $D \in \mathscr{D}(M, \mathscr{F})$. Then, there exists a vector field $W$ on $M$ such that $D=\left.\operatorname{ad} W\right|_{\mathscr{T}(M, \mathscr{F})}$. Moreover, $W$ is in $\mathscr{L}(M, \mathscr{F})$.

Proof. Take a distinguished coordinate neighborhood system $\left\{U_{\lambda}\right.$; $\left.\left(x_{1}^{\lambda}, \ldots, x_{p}^{\lambda}, y_{1}^{\lambda}, \ldots, y_{q}^{\lambda}\right)\right\}_{\lambda \in \Lambda}$ on $(M, \mathscr{F})$. Since $D$ is localizable, the derivation $D_{U_{\lambda}} \in D\left(U_{\lambda},\left.\mathscr{F}\right|_{U_{\lambda}}\right)$ can be defined for all $\lambda \in \Lambda$ in such a way that $\left.D(X)\right|_{U_{\lambda}}=D_{U_{\lambda}}\left(\left.X\right|_{U_{\lambda}}\right)$ for all $X \in \mathscr{T}(M)$. Then by Theorem 
3.2, there exists a unique vector field $W_{\lambda}$ on $U_{\lambda}$ such that $D_{U_{\lambda}}$ $=\left.\operatorname{ad} W_{\lambda}\right|_{\mathscr{T}\left(U_{\lambda}\right)}$ for any $\lambda \in \Lambda$. On the other hand, we have $\left.D_{U_{\lambda}}\right|_{U_{\lambda} \cap U_{\mu}}$ $=\left.D_{U_{\mu}}\right|_{U_{\lambda} \cap U_{\mu}}$, so $W_{\lambda}=W_{\mu}$ on $U_{\lambda} \cap U_{\mu}$. Hence there is a vector field $W$ on $M$ such that $W=W_{\lambda}$ on $U_{\lambda}$ for all $\lambda \in \Lambda$ and that $D=\left.\operatorname{ad} W\right|_{\mathscr{T}(M)}$. Moreover, we have $W \in \mathscr{L}(M)$, because $W_{\lambda} \in \mathscr{L}\left(U_{\lambda}\right)$ for all $\lambda \in \Lambda$.

Q. E. D.

Corollary 4.5. Let $D \in \mathscr{D}_{\mathscr{T}}(M, \mathscr{F})$ or $\mathscr{D}_{\mathscr{L}}(M, \mathscr{F})$. Then there exists a vector field $W$ on $M$ such that $D=\left.\operatorname{ad} W\right|_{\mathscr{T}(M, \mathscr{F})}$ or $=$ $\left.\operatorname{ad} W\right|_{\mathscr{L}(M, \mathscr{F})}$ respectively. Moreover, $W$ is in $\mathscr{L}(M, \mathscr{F})$.

Proof. Obvious for the case $D \in \mathscr{D}_{\mathscr{T}}(M)$. Let $D \in \mathscr{D}_{\mathscr{L}}(M)$. The restriction of $D$ to $\mathscr{T}(M)$ belongs to $\mathscr{D}(M)$. Then the assertion follows from Proposition 4. 4 and Lemma 4.1.

Q. E. D.

Then we get Main Theorem similarly as Theorem 3.4.

Theorem 4.6. ( $i)$ All derivations of $\mathscr{L}(M, \mathscr{F})$ are inner, that is, $\mathscr{D}_{\mathscr{L}}(M, \mathscr{F})=\operatorname{ad} \mathscr{L}(M, \mathscr{F}) \cong \mathscr{L}(M, \mathscr{F})$. Hence

$$
H^{1}(\mathscr{L}(M, \mathscr{F}) ; \mathscr{L}(M, \mathscr{F}))=0 .
$$

(ii) The derivation algebra of $\mathscr{T}(M, \mathscr{F})$ is naturally isomorphic to $\mathscr{L}(M, \mathscr{F})$, that is, $\mathscr{D}_{\mathscr{T}}(M, \mathscr{F})=\left\{\left.\operatorname{ad} W\right|_{\mathscr{T}(M . \mathscr{F})} ; W \in \mathscr{L}(M, \mathscr{F})\right\}$ $\cong \mathscr{L}(M, \mathscr{F})$. Hence

$$
H^{1}(\mathscr{T}(M, \mathscr{F}) ; \mathscr{T}(M, \mathscr{F})) \cong \mathscr{L}(M, \mathscr{F}) / \mathscr{T}(M, \mathscr{F}) .
$$

4. 4. Examples. Let $H^{1}=H^{1}(\mathscr{T}(M, \mathscr{F}) ; \mathscr{T}(M, \mathscr{F})) \cong \mathscr{L}(M$, $\mathscr{F}) / \mathscr{T}(M, \mathscr{F})$ for a foliated manifold $(M, \mathscr{F})$. In many cases, $H^{1}$ are of infinite dimension.

Proposition 4.7. Assume that there is a compact leaf $L$ of $\mathscr{F}$ such that there is a saturated neighborhood $U$ of $L$, which is a product foliation $D^{q} \times L$, where $D^{q}$ is a q-dimensional disk. Then, $H^{1}$ is of infinite dimension. 
Proof. Every leaf in $U$ is represented by a point of $D^{q}$. Let $f$ be a function supported in $D^{q}$. Then $f \circ \mathscr{L}(M, \mathscr{F}) \subset \mathscr{L}(M, \mathscr{F})$. Hence the assertion follows from Theorem 3.4. $\quad$ Q. E. D.

However, $H^{1}$ may be of finite dimension. Assume that $M$ is compact. J. Leslie [5] gives examples of $\operatorname{dim} H^{1}=0$, or 1: (i) an Anosov flow with an integral invariant for $\operatorname{dim} H^{1}=0$, and (ii) irrational flows on a two dimensional torus $T^{2}$ for $\operatorname{dim} H^{1}=1$. We can modify the latter to get a foliated manifold with $\operatorname{dim} H^{1}=n$ (for arbitrary $n<+\infty)$, that is, irrational flows on an $(n+1)$-dimensional torus $T^{n+1}$.

We have also other examples. Fukui and Ushiki [2] shows that $\operatorname{dim} H^{1}=2$ for the Reeb foliation on a 3 -shpere $S^{3}$. Further, Fukui [1] shows that the following: let $(M, \mathscr{F})$ be a Reeb foliated 3manifold, then $\operatorname{dim} H^{1}$ is finite, and equals to the number of generalized Reeb components.

\section{References}

[1] Fukui, K., On the homotopy type of some subgroups of Diff $\left(M^{3}\right)$, Japan. J. Math., 2(1976), 249-267.

[2] Fukui, K. and Ushiki, S., On the homotopy type of FDiff $\left(S^{3}, \mathscr{F}_{R}\right), J$. Math., Kyoto Univ., $\mathbb{1 5}$ (1975), 201-210.

[3] Kanie, Y., Cohomologies of Lie algebras of vector fields with coefficients in adjoint representations; Hamiltonian Case, Publ. RIMS, Kyoto Univ., 110 (1975), 737-762.

[4] Kanie, Y., Cohom ologies of Lie algebras of algebras of vector fields with coefficients in adjoint representations; Case of Classical Type, Publ. RIMS, Kyoto Univ., $\mathbb{1 1}$ (1975), 213-245.

[5] Leslie, J., A remark on the group of automorphisms of a foliation having a dense leaf, J. Differential Geometry, 7 (1972), 597-601.

[6] Reeb, G., Sur certaines propriétés topologiques des variétés feuilltées, Act. Sci. Ind., Hermann, Paris, 1183 (1952), 83-154.

[7] Takens, F., Derivations of vector fields, Compositio Math., 25 (1973), 151-158. 
